Check for updates

Cite this: RSC Adv., 2018, 8, 12933

Received 10th February 2018

Accepted 14th March 2018

DOI: $10.1039 / c 8 \mathrm{ra01305e}$

rsc.li/rsc-advances

\section{Preparation of open-porous stereocomplex PLA/ PBAT scaffolds and correlation between their morphology, mechanical behavior, and cell compatibility}

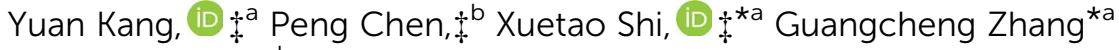 \\ and Chaoli Wang ${ }^{\mathrm{b}}$
}

For tissue engineering applications, it is essential that biodegradable scaffolds have accessible mechanical properties, high porosity, and good biocompatibility to support the formation of new tissues. In this study, we have prepared stereocomplex polylactide (sc-PLA) incorporated poly(butylene adipate-coterephthalate) (PBAT) scaffolds by non-solvent induced phase separation (NIPS). Also, we have characterized and compared the morphology, thermal, mechanical, and wettability properties as well as preliminary biocompatibility of scaffolds. The developed sc-PLA/PBAT scaffolds possess high porosity (>94\%), well-connected open microporous structures, accessible mechanical properties, and excellent water permeability. As the content of PBAT increased, the average diameter of the SC-PLA/PBAT scaffolds decreased while the mechanical properties improved. The tensile strength was improved to 3.8 MPa while the neat PLA scaffold was $0.3 \mathrm{MPa}$, and the elongation of the scaffold was six times higher than neat PLA scaffold. Fibroblasts cells seeded on the structure maintained phenotypic shape, and the developed scaffold structure was observed to be highly capable of supporting the cell attachment and proliferation.

\section{Introduction}

Poly(lactic acid) (PLA) bioplastic is a fully biodegradable polymer with excellent biocompatibility and processing properties. It is non-toxic to the human body and the environment. It has three isomeric forms, namely, poly(L-lactide) (PLLA), poly(D-lactide) (PDLA), and poly(racemic-lactide) (PDLLA), which display a wide variety of properties. ${ }^{1-3}$ Macromolecules with identical chemical composition but different stereochemical configuration of repeating units are able to form intermolecular complexes called stereocomplexes. An example of such specific complex is stereocomplex polylactide (sc-PLA) formed from an equimolar mixture of enantiomeric PLLA and PDLA. Stereocomplexation can occur between PLLA and PDLA either in solution or during the melting process. The sc-PLA materials exhibit relatively more desirable mechanical characteristics, thermal resistance, and biological degradability. Therefore, scPLA has attracted the attention of many researchers in recent

${ }^{a}$ Department of Applied Chemistry, School of Natural and Applied Sciences, Northwestern Polytechnical University, Xi'an 710129, P. R. China. E-mail: shixuetao@nwpu.edu.cn

${ }^{b}$ Department of Pharmaceutical Analysis, School of Pharmacy, Fourth Military Medical University, Xi'an, 710032, China

$\$$ These authors contributed equally to this study. years and expanded its use in tissue engineering or imitating extracellular matrix as functional materials for cell growth. ${ }^{4-8}$

In recent years, although porous and highly-interconnected PLA scaffolds are expected to be a potential alternative scaffold for tissue engineering application, there are also certain drawbacks regarding its performance, such as low elongation, poor heat resistance, and weak impact-resistance strength. ${ }^{\mathbf{9 - 1 3}}$ As a result, there is a need to modify them for certain applications. Till now, some developments in the modification of PLA including copolymerization, blending, compounding, and additives were highlighted to improve its relevant properties as used for scaffold materials. ${ }^{\mathbf{1 4 - 1 7}}$ Nanocomposites is another technology for the polymer modification with enhanced mechanical properties, antibacterial property and other multifunctionalizations. ${ }^{18,19}$ Patrício et al. toughened PLA scaffold by introducing high-toughness polycaprolactone (PCL) into scaffold matrix, ${ }^{20}$ resulting in PLA/PCL composite scaffolds that possess better mechanical properties and biocompatibility. Also, Scaffaro et al. $^{21}$ prepared porous PLA/polyethyleneglycol (PEG) scaffold, which showed lower elongation and enhanced hydrophilic properties, due to the abundant hydrophilic groups and flexible PEG molecule. Haaparanta et al. ${ }^{22}$ fabricated a chitosan-coated PLA scaffold by modification of the pore surface using a chitosan coating process. The results show that the chitosan-coated PLA scaffold has improved hydrophilicity and 
better mechanical properties as compared to the untreated PLA scaffold. In our former work, ${ }^{23}$ the microcellular PLA/PBAT blends was prepared by batch foaming process with supercritical carbon dioxide, which found the addition of PBAT induced the open-cell structure of cell morphology instead of the typical close-cells of pure PLLA. This prepared foam had a very lower porosity and could not meet requirement for potential application in tissue engineering.

Furthermore, the existing PLA modification methods do not fully meet the needs of tissue engineering. Tissue engineering scaffolds of different organs require a specific performance of the scaffold material. In the preparation of tissue engineered cartilage, the scaffold material needs good elasticity for physiological function and pore formation for materials exchange of cells. As we know, PBAT is a biodegradable, flexible material. It has a high elongation at break, as well as excellent processing properties. The carbonyl in PBAT molecular chains makes PBAT compatible or partially compatible with PLA during the blending process. ${ }^{24}$ Therefore, PBAT has been widely studied for modifying fragile polymers. Signori et $a .^{25}$ studied the compatibility, crystallization, and tensile properties of PLA/ PBAT blends in different proportions produced by melt blending in the presence of dicumyl peroxide. Lins et al. ${ }^{26}$ reported interesting work of PLA/PBAT blends with phosphonium ionic liquids as new compatibilizing agents, resulting in enhanced Young's modulus without loss of the fracture properties. The results demonstrate that PBAT has a significant effect on toughening of PLA. Al-Itry et al. ${ }^{27,28}$ first investigated the mechanisms and conditions governing thermal degradation of PLA/PBAT blends by thermal analysis and size-exclusion chromatography. Although PBAT has attracted more attrition and been used for toughening various polymers, till date, there has not been much research reported to prepare porous PLA/ PBAT scaffolds.

In this work, we choose poly(butylene adipate-co-terephthalate) (PBAT) as toughness modifier for the PLA scaffold matrix. The aim of this research is to manufacture novel sc-PLA/ PBAT scaffolds by non-solvent phase separation technology, with high porosity and well-connected open porous micropores structures, for use in tissue engineering cartilage. The hybrid scaffolds are compared with neat PLA and PBAT porous scaffolds by determining the microstructure, water uptake abilities, mechanical properties, and cell compatibility and attachment with adult bovine fibroblasts aimed to ascertain the suitability for tissue engineering applications.

\section{Experiment}

\subsection{Materials}

In this work, the selected PLLA and PDLA were supplied by Corbion Purac (The Netherlands) with the trade name L175 $\left(M_{\mathrm{W}} \sim 150000 \mathrm{~g} \mathrm{~mol}^{-1},>99 \% \mathrm{~L}\right.$ isomer $)$ and D070 $\left(M_{\mathrm{w}} \sim\right.$ $70000 \mathrm{~g} \mathrm{~mol}{ }^{-1},>99 \% \mathrm{D}$ isomer), respectively. PBAT was supplied by BASF under the trade mark of Ecoflex (density: $1.26 \mathrm{~g} \mathrm{~cm}^{-3}$; melt index: $4.5 \mathrm{~mL} / 10 \mathrm{~min}$ at $190{ }^{\circ} \mathrm{C}$; and melt temperature: $110-120^{\circ} \mathrm{C}$ ). Sodium dodecylbenzenesulfonate (SDBS) and dichloromethane was bought from Sinopharm
Chemical Reagent Co., Ltd, Shanghai, China. Fibroblasts cell line L929 was purchased from China Center for Type Culture Collection (CCTCC) and maintained in RPMI medium 1640 (Gibco, CA) supplemented with $10 \%$ fetal bovine serum (Gibco, CA). Cells were incubated in a $5 \% \mathrm{CO}_{2}$ humidified incubator (Thermo Scientific, GER) at $37{ }^{\circ} \mathrm{C}$, and collected using $0.05 \%$ trypsin EDTA (YaxinBio, CHN) following the specified incubation period.

\subsection{Preparation of Sc-PLA/PBAT matrices}

Polymers of PLLA, PDLA, and PBAT (total weight $=2 \mathrm{~g}$ ) were dissolved in dichloromethane solvent $(40 \mathrm{~mL})$ at room temperature. The sc-PLA/PBAT weight ratio was kept in the range of $100: 0$ to $0: 100$, including $100: 0,20: 80,40: 60$, $50: 50,60: 40$, and $0: 100$. And then the solutions were stirred continuously with a magnetic stirrer for 5 hours. In this work, the PLLA/PDLA weight ratio of PLA was fixed at $5: 5$.

\subsection{Fabrication of sc-PLA/PBAT scaffolds}

The sc-PLA/PBAT scaffolds were fabricated by the double emulsion method and the schematic of the preparation process is shown in Fig. 1. SDBS (0.25 g) was dissolved as anionic molecule active agent in water phase solvent. PLA and PBAT (2 g) with different weight ratio were dissolved in dichloromethane by mechanical stirring for 5 hours and the resultant PLA/PBAT solution $(40 \mathrm{~mL})$ was prepared as the oil phase. The water phase/oil phase ratio ranged from $0.4 / 1(\mathrm{v} / \mathrm{v})$ to $0.9 / 1(\mathrm{v} / \mathrm{v})$. Thereafter, the two solutions were mixed completely by ultrasonic cell disruptor (20 $\mathrm{min}$ ) for homogeneous dispersion. The obtained sc-PLA/PBAT composites were centrifuged for $5 \mathrm{~min}$ at a speed of $9000 \mathrm{rpm}$. Afterwards, the composites were kept in a drying oven at $40{ }^{\circ} \mathrm{C}$ to volatilize dichloromethane solvent and water for 72 hours.

\subsection{Characterization of SC-PLA/PBAT scaffolds}

Differential scanning calorimetry (DSC), X-ray diffraction (XRD) and Fourier-transform infrared spectroscopy (FT-IR). The DSC analysis was carried out by Mettler differential scanning calorimetry (DSC1, USA). The samples were heated from $25^{\circ} \mathrm{C}$ to $250{ }^{\circ} \mathrm{C}$ at $10{ }^{\circ} \mathrm{C} \mathrm{min}^{-1}$. All processing was protected with nitrogen. The glass transition temperature and the melting temperature were acquired from the DSC curves. XRD curves of porous scaffold membranes were obtained by an X-ray diffraction at $4^{\circ} \mathrm{min}^{-1}$ in the scanning angle range of $2 \theta=10-50^{\circ}(\mathrm{D} 2$ Phaser, Brucker, Germany). Infrared spectra of PLA/PBAT blend composites were also analyzed by FT-IR (TENSOR 27, Brucker, Germany). FT-IR spectra were measured using $\mathrm{KBr}$ as background in the range of 1000 to $500 \mathrm{~cm}^{-1}$ with a wavelength resolution of $4 \mathrm{~cm}^{-1}$.

Surface morphology examination. The surface morphology of the scaffolds was examined using scanning electron microscopy with an accelerating voltage of $10 \mathrm{kV}$ (SEM, Tescan Vega3, Czech). For the measurements, the scaffolds were coated with gold using a sputter coater. During the process of gold coating, the current was $50 \mathrm{~mA}$ and coating duration was $90 \mathrm{~s}$. 


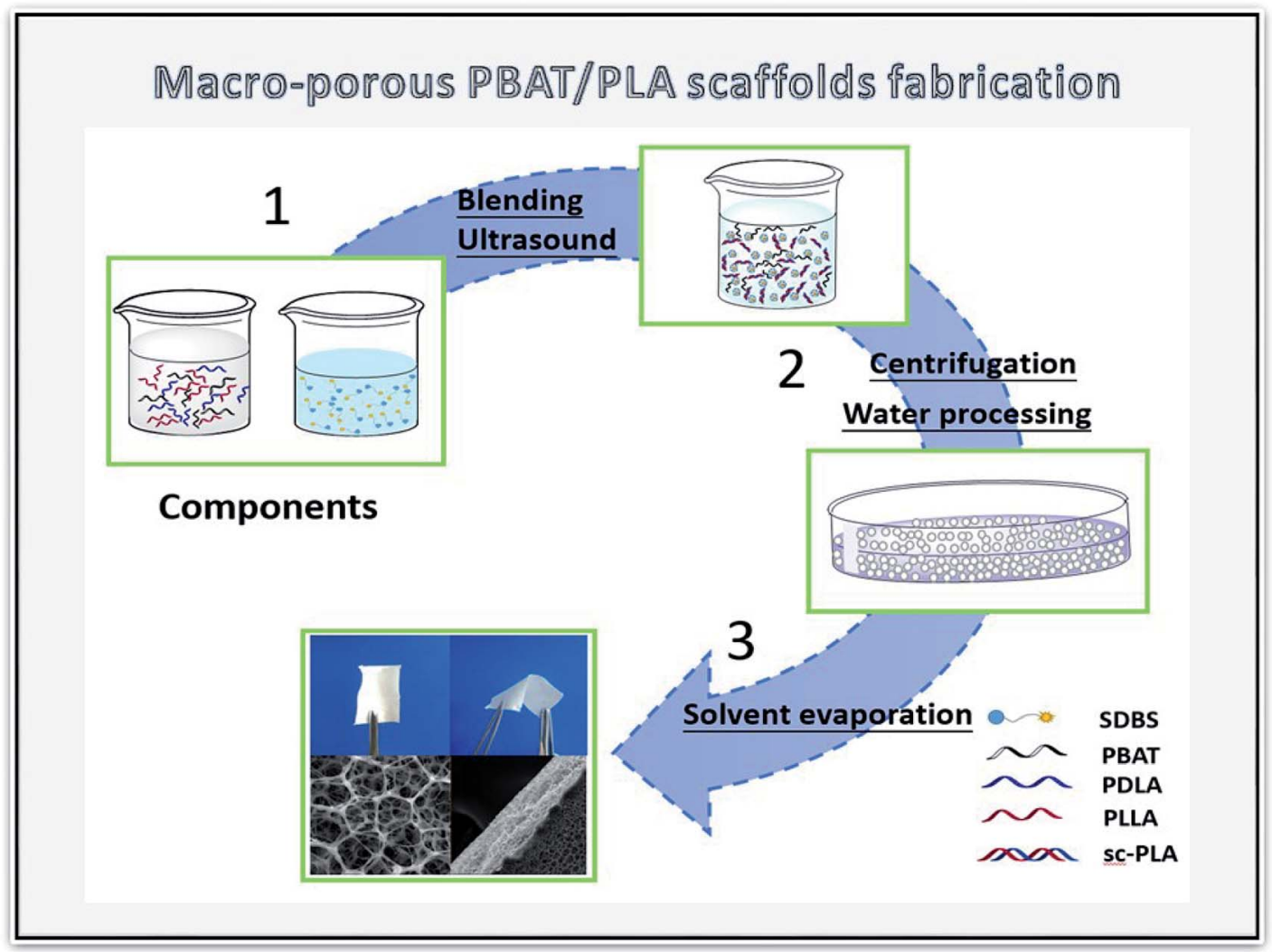

Fig. 1 Schematic of the preparation of biomimetic sc-PLA/PBAT scaffolds by NIPS process.

Porosity measurement. Porosity of the sc-PLA/PBAT scaffolds were measured by a mercury porosimeter (AutoPore IV 9500 V1.08, Micrometritis Instrument Co., Nacross, GA). The scaffold samples were cut into $10 \times 10 \times 0.5 \mathrm{~mm}^{3}$ (length $\times$ width $\times$ thickness) rectangular solid shapes, weighed, and placed in the cup of the penetrometer, which was closed by tightening the cap. Mercury filling pressure was 0.5 psia, while equilibration time was $10 \mathrm{~s}$.

Wettability. Water uptake of porous scaffolds was measured by immersing them in phosphate buffered solution (PBS, $0.01 \mathrm{M}, \mathrm{pH}=7.4)$. Firstly, cylinder-shaped samples of each scaffold with diameter of $10 \mathrm{~mm}$ and height of $0.5 \mathrm{~mm}$ were dried in a vacuum oven, then weighed and recorded as $W_{\mathrm{s}}$. Next, they were soaked in PBS and kept at $37{ }^{\circ} \mathrm{C}$ at a constant temperature bath oscillator for 24 hours. Finally, the saturated scaffolds were removed from the solution and held over a filter paper to remove excess water on the surface, and then weighed and recorded. The water uptake of the scaffolds was calculated gravimetrically according to eqn (1).

$$
\text { Water uptake }=\frac{W_{\mathrm{s}}-W_{\mathrm{o}}}{W_{\mathrm{o}}} \times 100 \%
$$

where $W_{\mathrm{s}}$ is the mass of the swollen material, and $W_{\mathrm{o}}$ is the initial dry mass.

To investigate the hydrophilicity of scaffolds, the dynamic contact angle of scaffolds was measured by a Data Physics OCA20 device. The sample scaffolds were cut into flat pieces $\left(2 \times 1 \times 0.05 \mathrm{~cm}^{3}\right)$ and placed on a glass microscope slide. An ultrapure water droplet size of $3 \mu \mathrm{L}$ was placed on the sample surface at room temperature. All contact angle measurements were performed in five replicates.

Mechanical performance. Sample scaffolds were cut into 50 $\times 20 \mathrm{~mm}$ rectangle samples with the thickness of about 0.05 $\mathrm{mm}$, and the effective length of tensile samples was $20 \mathrm{~mm}$. The uniaxial tensile test was carried out at a tensile rate of 10 $\mathrm{mm} \mathrm{min}^{-1}$ by the Instron 3340 tension tester made in USA. The average tensile strength and elongation-at-break were measured from at least five samples.

\section{Cellular compatibility test}

MTT assays. Survival rate of fibroblast L929 cells cultured on 24-well plates with sample scaffolds was determined by 3-(4,5dimethylthiazol-2-yl)-2,5-diphenyltetrazolium bromide (MTT) assay. Cells were cultured for 12, 24, 72, and 96 hours. For quantitation, $80 \mu \mathrm{L}$ MTT was added into each well and incubated at $37{ }^{\circ} \mathrm{C}$ for $4 \mathrm{~h}$. The medium was removed and $800 \mu \mathrm{L}$ dimethylsulfoxide was added, and then $150 \mu \mathrm{L}$ solution was extracted to 96-well after shaking for $10 \mathrm{~min}$. The absorbance value was measured using a multi-well Spectrophotometer at $490 \mathrm{~nm}$.

Flow cytometry (FCM). L929 cells were collected at a concentration of $2 \times 10^{4}$ cells per $\mathrm{mL}$, and were incubated with sample scaffolds in a 6-well plate for $48 \mathrm{~h}$ at $37^{\circ} \mathrm{C}$. Simultaneously, cells without scaffolds were cultured as a negative control. Then, cells were washed with PBS, fixed with $75 \%$ ethanol, and stained 

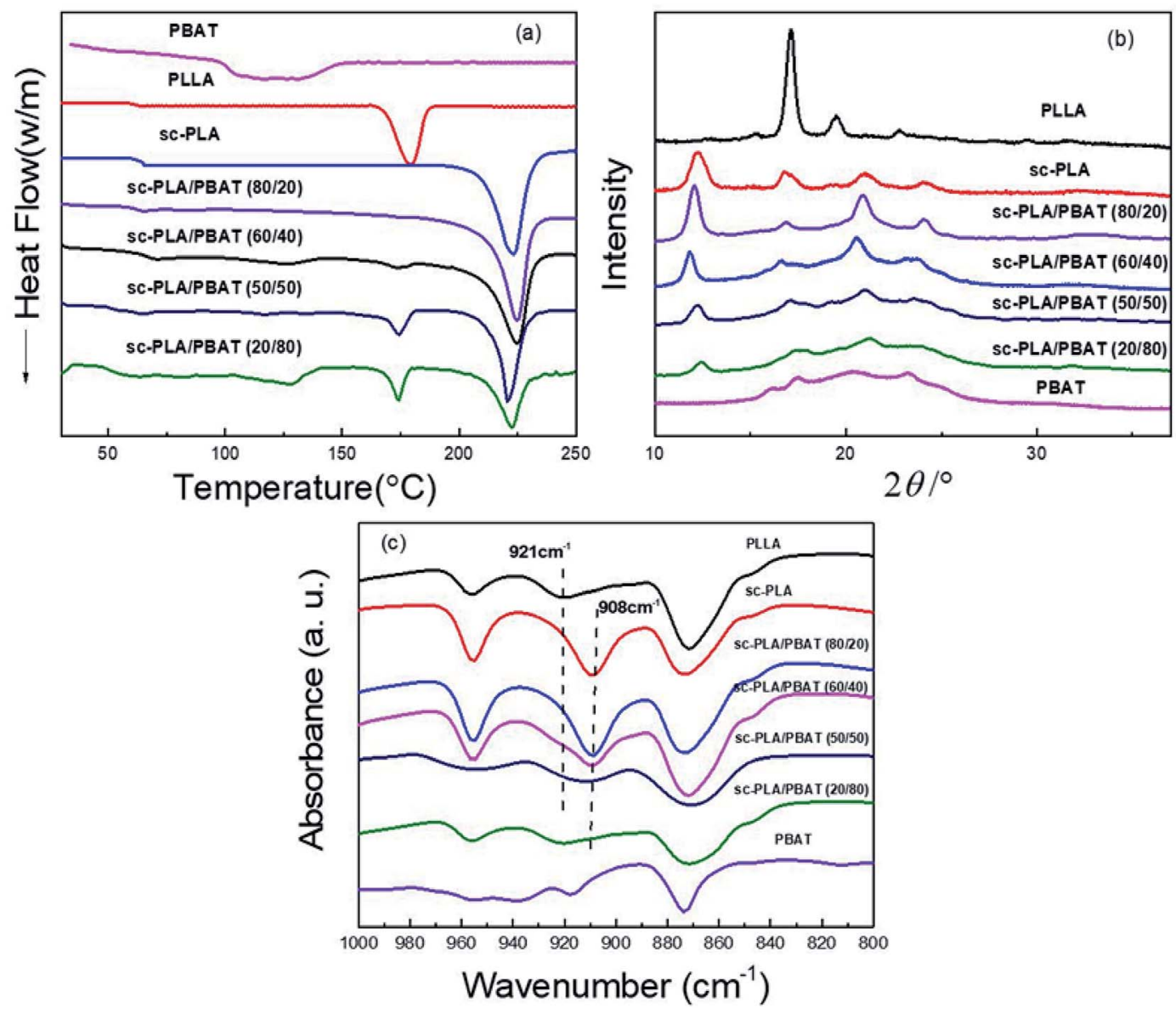

Fig. 2 Characterization of neat PLLA, sc-PLA, PBAT and sc-PLA/PBAT scaffolds: (a) DSC first heating curves (heating rate: $10^{\circ} \mathrm{C}$ min ${ }^{-1}$ ); (b) XRD patterns; (c) FI-TR patterns.

with propidium iodide in the dark for $15 \mathrm{~min}$, in sequence. Finally, a Beckman FC500 flow cytometer was used to detect cell cycle.

Cell culture of fibroblasts on scaffolds. Sample scaffolds were placed in 6-well plates and seeded with $2 \mathrm{~mL}$ cell suspension containing $1 \times 10^{5}$ L929 cells. After 36 hours, the cell-seeded samples were removed from the media and gently rinsed with phosphate-buffered solution. L929 cells cultured on the scaffolds were then fixed with $2.5 \%$ glutaraldehyde solution for 12 hours at $4{ }^{\circ} \mathrm{C}$. After removing the fixative, the scaffolds were dehydrated for $15 \mathrm{~min}$ with ethanol at a series of concentrations (30, 50, 70, 80, 90, and 100\%). Afterwards, the samples were dried for 12 hours and coated with platinum for 40 seconds prior to SEM examination at an accelerating voltage of $5 \mathrm{kV}$.

Statistical analysis. The data are expressed as the means \pm SD. All experiments were repeated at least three times and the

Table 1 Melting and crystallization parameters of sc-PLA/PBAT blends ${ }^{a}$

\begin{tabular}{|c|c|c|c|c|c|c|c|}
\hline Samples & $T_{\mathrm{g}}\left({ }^{\circ} \mathrm{C}\right)$ & $T_{\mathrm{hm}}\left({ }^{\circ} \mathrm{C}\right)$ & $T_{\mathrm{sm}}\left({ }^{\circ} \mathrm{C}\right)$ & $\Delta H_{\mathrm{hm}}\left(\mathrm{J} \mathrm{mol}^{-1}\right)$ & $\Delta H_{\mathrm{sm}}\left(\mathrm{J} \mathrm{mol}^{-1}\right)$ & $X_{\mathrm{hc}}(\%)$ & $X_{\mathrm{sc}}(\%)$ \\
\hline PLLA & 62.4 & 175.0 & & 24.3 & & 26.1 & - \\
\hline sc-PLA & 63.1 & - & 223.0 & - & 34.7 & - & 24.44 \\
\hline sc-PLA/PBAT $(60 / 40)$ & 62.7 & 175.2 & 225.1 & 1.7 & 27.0 & 2.28 & 23.77 \\
\hline sc-PLA/PBAT (50/50) & 59.7 & 174.3 & 221.9 & 3.8 & 22.1 & 5.11 & 19.45 \\
\hline sc-PLA/PBAT (20/80) & 54.2 & 174.4 & 222.6 & 5.0 & 19.4 & 6.72 & 17.08 \\
\hline
\end{tabular}

${ }^{a} T_{\mathrm{g}}$ is the glass transition temperature; $T_{\mathrm{hm}}$ and $T_{\mathrm{sm}}$ are the melting peaks of PLA homopolymer and stereocomplexes, respectively; $\Delta H_{\mathrm{hm}}$ and $\Delta H_{\mathrm{sm}}$ are the enthalpy of homopolymer melting and stereocomplexes melting, respectively; the crystallinity of PLLA calculated by the equation: $X_{\mathrm{c}}(\%)=$ $\Delta H_{\mathrm{m}} / \Delta H_{\mathrm{m}}^{0}\left(1-W_{\mathrm{f}}\right), \Delta H_{\mathrm{m}}^{0}$ equals $93 \mathrm{~J} \mathrm{~g}^{-1}$ for $100 \%$ crystalline of PLA homopolymer, ${ }^{7}$ and equals $142 \mathrm{~J} \mathrm{~g}^{-1}$ (ref. 8) and $114 \mathrm{~J} \mathrm{~g}^{-1}$ (ref. 30 ) for $100 \%$ PLA stereocomplex and PBAT, respectively. $W_{\mathrm{f}}$ represents the weight percentage of the added PBAT. 

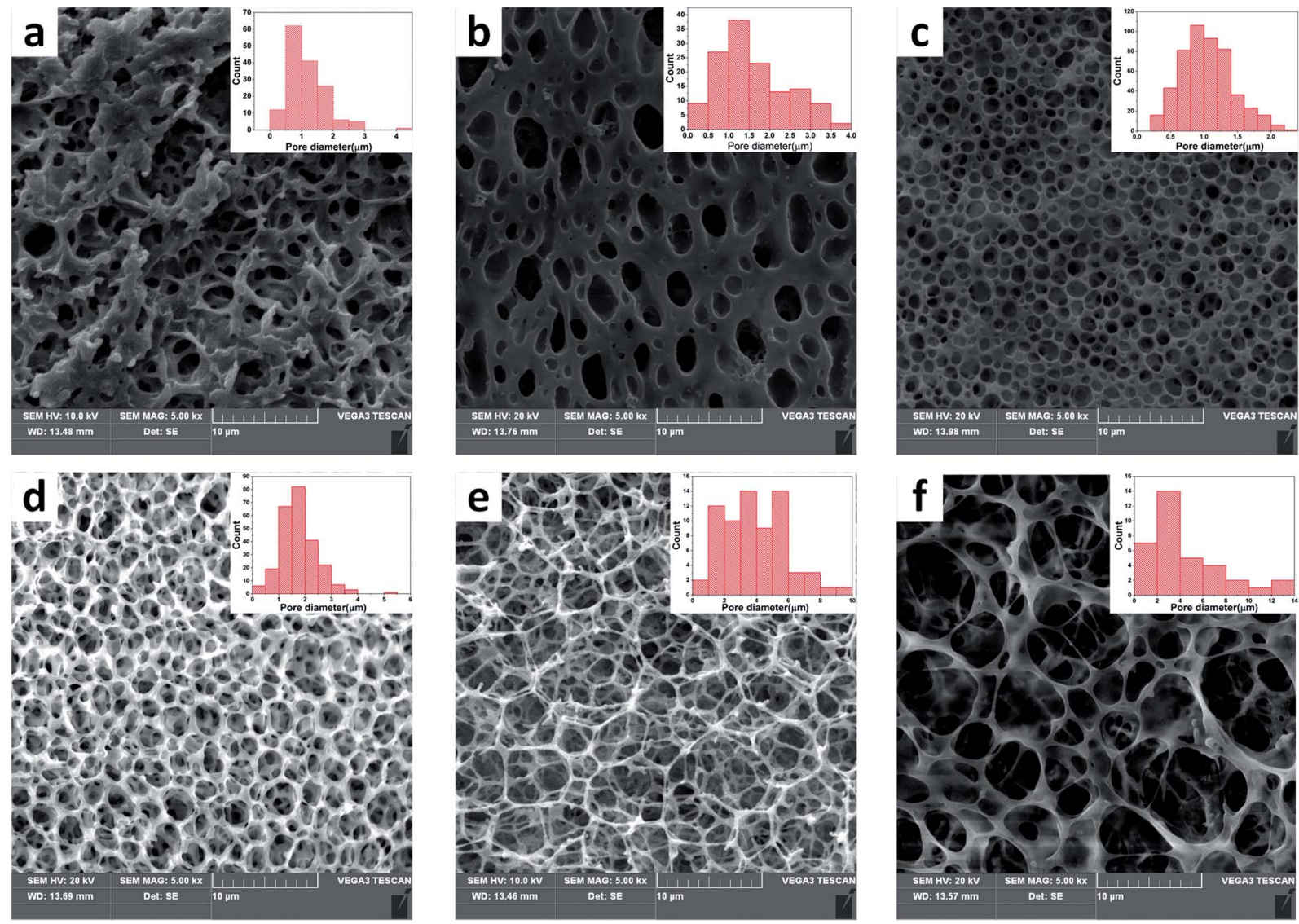

Fig. 3 SEM micrographs and pore distribution diagrams of SC-PLA/PBAT (70/30) scaffolds prepared with different water/dichloromethane ratios: (a) $w / o=0.4 / 1$; (b) $w / 0=0.5 / 1$; (c) $w / o=0.6 / 1 ;$ (d) $w / o=0.7 / 1$; (e) $w / o=0.8 / 1$; (f) $w / o=0.9 / 1$.

test was performed to test the significance of the results. All data were considered statistically significant at $P<0.05$.

\section{Results and discussion}

\subsection{Influence of PBAT on the PLA stereocomplexation}

With respect to PLA, stereocomplexed materials can be prepared by blending optically active and isotactic PLLA with its enantiomeric polymer, PDLA. Stereocomplexed PLA materials have higher mechanical performance compared to either PLLA or PDLA. ${ }^{29}$ In order to investigate the thermal performance and crystallization caused by stereocomplexation and different matrices, we prepared neat PLLA, neat PBAT, sc-PLA (by blending same weight PLLA and PDLA in solution) and sc-PLA/ PBAT scaffolds with same preparation process $(\mathrm{w} / \mathrm{o}=0.8 / 1)$. Fig. 2a shows the first heating DSC thermograms of the solution-casting sample scaffolds. The related glass transition temperature, melting temperature, and the crystallinity of each specimen are recorded in Table 1 . Here, the glass transition temperatures of neat PLA and PBAT are around $63{ }^{\circ} \mathrm{C}$ and $-34{ }^{\circ} \mathrm{C},{ }^{26}$ respectively. The value of $T_{\mathrm{g}}$ decreases slightly with increasing PBAT content. The melting peaks of pure PLLA matrix is observed at $175-180{ }^{\circ} \mathrm{C}$. Meanwhile, the melting peak of sc-PLA scaffold is seen at $220-225{ }^{\circ} \mathrm{C}$, which is almost $45{ }^{\circ} \mathrm{C}$ higher than that of pure PLLA, suggesting that stereocomplexation mechanism between PLLA and PDLA molecules. The benefits of enhanced thermal stability and mechanical properties is strongly related to the intermolecular force of sc-PLA. Another scaffold of PBAT possesses melt peak from $121^{\circ} \mathrm{C}$ to $131^{\circ} \mathrm{C}$. With the introduction of PBAT into PLLA/ PDLA blends, the DSC curves of sc-PLA/PBAT blends present an additional new melting peak (173-177 $\left.{ }^{\circ} \mathrm{C}\right)$ referred to homopolymer PLLA or PDLA when the PBAT concentration is above $20 \%$. In addition, the increasing content of PBAT in blend leads to lower stereocomplexation crystallinity and higher homogeneous crystallinity as recorded in Table 1 . The result illustrates that the addition of PBAT restrains stereocomplexation of PLA. In other words, the second immiscible PBAT would lead to less chances for the intermolecular hydrogen bonding between PLLA and PDLA.

The similar phenomenon was observed in XRD patterns. The XRD patterns of PLLA, sc-PLA, PBAT, and sc-PLA/PBAT scaffolds are displayed in Fig. 2b. PLLA clearly exhibited some diffraction peaks at $2 \theta=15.2,17.1,19.4$, and $22.8^{\circ}$, assigned to characteristic peaks of $\alpha$-form crystals crystallized in a pseudoorthorhombic unit cell. ${ }^{31}$ Whereas, the characteristic peaks of sc-PLA, with values of $12.0,21.0$, and $24^{\circ}$, corresponding to scPLA, crystallized in a triclinic unit cell, in which the $\mathrm{L}^{-}$and $\mathrm{D}^{-}$ lactide segments are packed parallel in a helical conformation. ${ }^{32}$ Four diffraction peaks close to $16.1,17.5 .5,20.6$ and $23.1^{\circ}$ 
were observed in the pattern of the PBAT scaffold, thus indicating the presence of $\alpha$-form crystalline structure. However, there is an additional sc-form characteristic peak at $11.8^{\circ}$ of scPLA/PBAT scaffolds XRD pattern which presents lower intensity than sc-PLA at same angle of $11.8-12.0^{\circ}$. With more PBAT, the degree of PLA stereocomplexation is decreased. This result is consistent with DSC analysis.

As shown in Fig. 2c, pure PLLA presents a characteristic FTIR absorption peak at $921 \mathrm{~cm}^{-1}$. The peak refers to $\alpha$-form crystals crystallized in a pseudo-orthorhombic unit cell of homogeneous PLA. ${ }^{33}$ However, the peak of sc-PLA is emerged at $908 \mathrm{~cm}^{-1}$, assigned to characteristic peaks of $\beta$-form crystals in a triclinic unit cell. ${ }^{34}$ As the increasing content of PBAT into scPLA, the characteristic peak of Sc-PLA become less obvious and the peak of homogeneous PLA emerges. The FT-IR results again confirm the similar conclusion that more PBAT in the PLLA/ PDLA blend would restrain the PLA stereocomplexation, which is also indicated by DSC and XRD analysis.

\subsection{Pore characteristics of porous scaffolds}

The open-porous scaffolds were fabricated by NIPS with a double emulsion process. The structure of pores began to take shape with the continuous volatilization of solvent dichloromethane and took a final form after the sublimation of freeze water. Therefore, the ratio ( $\mathrm{v} / \mathrm{v})$ of water and dichloromethane has a critical impact on the pore structure in scaffold. Fig. 3 shows the microstructure and pore diameter distribution graphs of sc-PLA/PBAT scaffolds fabricated based on different water/dichloromethane ratio (v/v) ranging from 0.4/1-0.9/1 (v/v) with same preparation conditions with a ratio of sc-PLA and PBAT is 70/30. As summarized in Table 2, the mean pore diameter became larger (ranging from 0.76 to $4.52 \mu \mathrm{m}$ ) and the open porosity increased with the increasing ratio. The scaffolds exhibit small, round, and closed porous structures at the ratio of less than $0.6: 1$. However, when the ratio is greater than 0.6 / 1 , the blend scaffolds present open-porous structures and the thickness of pore walls decreased correspondingly. For the scaffold with ratio $0.8 / 1$, hexagonal and nanofibers pore walls structure were observed obviously, and the open-porous scaffold possessed extremely high porosity of 94.1\%. Further increasing the water/dichloromethane ratio led to broken structures, non-uniform pore size, and wall thickness. Therefore, this result indicates that the pore structure formation of sc-PLA/PBAT scaffolds is extremely related with the water/ dichloromethane ratio in NIPS process. The pore size of the scaffolds varies with the content of the non-solvent, where higher ratio leads to a larger pore size. In order to build predesigned interconnected scaffolds with high open-porous

Table 2 Structural parameters of Sc-PLA/PBAT scaffolds prepared with different water/dichloromethane ratios

\begin{tabular}{lllllll}
\hline Samples & $0.4 / 1$ & $0.5 / 1$ & $0.6 / 1$ & $0.7 / 1$ & $0.8 / 1$ & $0.9 / 1$ \\
Porosity $(\%)$ & 34.6 & 61.2 & 88.0 & 92.6 & 94.1 & 93.5 \\
Surface area $\left(\mathrm{m}^{2} \mathrm{~g}^{-1}\right)$ & 2.04 & 3.17 & 7.22 & 9.43 & 10.61 & 9.21 \\
Average diameter $(\mu \mathrm{m})$ & 0.76 & 1.58 & 1.04 & 1.76 & 3.96 & 4.52
\end{tabular}

porosity and uniform architecture, we selected water/ dichloromethane ratio of $0.8 / 1$ to prepare further scaffolds.

Because the stereocomplexation of PLA promotes formation of sc-form crystals leading to stronger intermolecular force, we further investigated the effect of stereocomplexation on the microstructure of PLA/PBAT scaffolds. Fig. 4 shows a series of SEM images for various open-porous scaffolds fabricated from PLLA/PBAT, sc-PLA/PBAT, and PDLA/PBAT, where the quality ratio of PLA and PBAT is $80: 20$ and the weight-average molecular weight of PLLA and PDLA is $M_{\mathrm{w}}=1.5 \times 10^{5} \mathrm{~g}$ $\mathrm{mol}^{-1}$ and PDLA $M_{\mathrm{w}}=7.0 \times 10^{4} \mathrm{~g} \mathrm{~mol}^{-1}$, respectively. Although all the scaffolds present highly open porous microstructures, we could observe some microstructural difference of pore and walls structures among the three sample scaffolds. From can be seen at Fig. $4 \mathrm{a}^{\prime}$ and $\mathrm{c}^{\prime}$, the pore sizes of PLLA/PBAT and PDLA/PBAT scaffolds were observed to be nonuniform and the pore walls were asymmetric, even broken at a few places, particularly where there was still agglomeration of imporous matrix in PDLA/PBAT scaffold. However, the scaffold base on sc-PLA/ PBAT presented sexangular pore structure and uniform walls thickness of about $1 \mu \mathrm{m}$ (shown at Fig. $4 \mathrm{~b}$ and $\mathrm{b}^{\prime}$ ). The phenomenon can probably be attributed to the strong intermolecular forces of sc-PLA molecules. Furthermore, two structure types, $\alpha$-form crystal based on PLLA or PDLA and sc-form crystal have contributed to different microstructure. Therefore, the structural analysis of the three sample scaffolds indicates
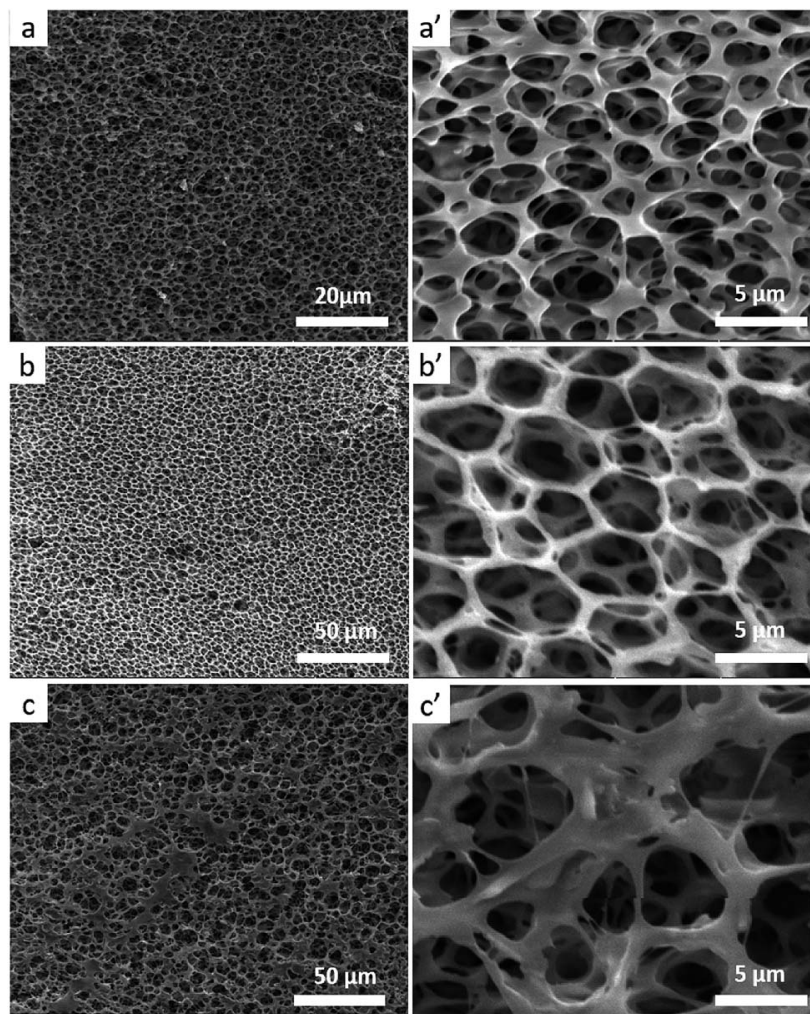

Fig. 4 SEM micrographs of scaffolds, (a) PLLA/PBAT (80/20) scaffold, $\times 2000$; (a') PLLA/PBAT scaffold (80/20), ×5000; (b) sc-PLA/PBAT (80/ 20) scaffold, $\times 2000$; (b') sc-PLA/PBAT (80/20) scaffold, $\times 5000$; (c) PDLA/PBAT (80/20) scaffold, $\times 2000$; (c') PDLA/PBAT (80/20) scaffold, $\times 5000$. 

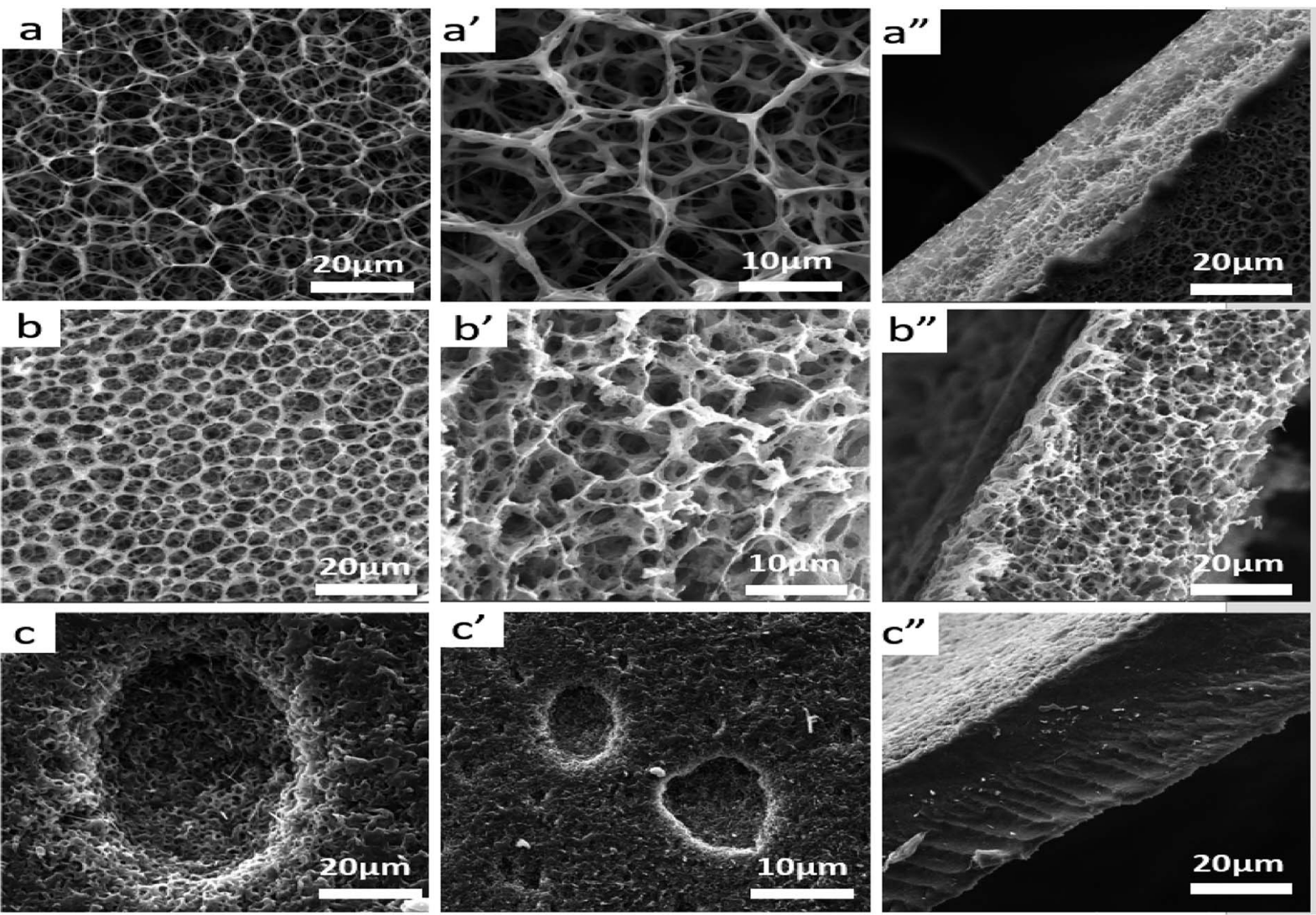

Fig. 5 SEM micrographs of scaffolds, (a) plane surface of sc-PLA scaffold, $\times 2000$; (a') plane surface of sc-PLA scaffold, $\times 5000$; (a") fracture surface of sc-PLA scaffold in thickness direction, $\times 5000$; (b) plane surface of sc-PLA/PBAT scaffold (50/50), $\times 2000$; (b') plane surface of sc-PLA/ PBAT (50/50) scaffold, $\times 5000$; (b") fracture surface of sc-PLA/PBAT (50/50) scaffold in thickness direction, $\times 5000$, scaffolds were prepared of 50/50 (w/w); (c) plane surface of PBAT scaffold, $\times 2000$; (c') plane surface of PBAT scaffold, $\times 5000$; (c") fracture surface of PBAT scaffold in thickness direction, $\times 5000$.

that the stereocomplexation of PLA promotes the formation of uniform and more opened porous scaffolds.

In literature, PLA has high strength and modulus (50-70 $\mathrm{MPa}$ ) but it is brittle (elongation at break is $3.8 \%$ ). However, PBAT is a kind of flexible and tough polymer with an elongation at break $710 \%{ }^{35}$ Therefore, the toughness of composite scaffolds would be improved by adding PBAT in to PLA matrix. Besides, the pore structures and mechanical properties of the scaffolds are affected by the content of PBAT in the matrix. SEM micrographs of plane and fractured surfaces of sc-PLA, sc-PLA/ $\operatorname{PBAT}(w / w=50 / 50)$, and PBAT scaffolds are shown in Fig. 5. The sc-PLA scaffold represents highly open porous structure with average pore size of about $9 \mu \mathrm{m}$, whereas the average pore size of sc-PLA/PBAT $(\mathrm{w} / \mathrm{w}=50 / 50)$ decreases by about $4 \mu \mathrm{m}$ (shown in Table 3). Furthermore, when the scaffold is fabricated of pure PBAT, the porous structure can be only observed at the surface of the scaffold. Data of porosity measurements presented in Table 3 indicate the porosity of scaffolds tend to decrease with increasing PBAT content, and it reduce to $2.56 \mathrm{~m}^{2} \mathrm{~g}^{-1}$ when the scaffold was prepared by neat PBAT. The result is probably due to the elasticity of PBAT would lead to pores shrinkage of scaffold during the phase separation processing. On the other hand, rapid shrinking of PBAT promotes water agglomeration, resulting in a small number of larger closed holes on the surface of the PBAT scaffold.

\subsection{Mechanical properties}

The stress-strain curves for the mechanical properties are shown in Fig. 6a. For the fragile and high porosity PLA scaffold, the tensile strength and elongation at break is very low. The scaffold materials show evident characteristic of brittle rupture when the ratio of PLA/PBAT is lower than 50/50. However, the

Table 3 Structural parameters of Sc-PLA/PBAT scaffolds prepared with different mass ratios

\begin{tabular}{llllll}
\hline Samples & $100 / 0$ & $80 / 20$ & $60 / 40$ & $50 / 50$ & $20 / 80$ \\
Porosity $(\%)$ & 93.5 & 91.2 & 89.0 & 87.6 & 46.1 \\
Surface area $\left(\mathrm{m}^{2} \mathrm{~g}^{-1}\right)$ & 9.97 & 9.33 & 10.12 & 11.03 & 4.11 \\
Average diameter $(\mu \mathrm{m})$ & $8.86 \pm 1.03$ & $5.72 \pm 0.85$ & $4.47 \pm 0.83$ & $3.96 \pm 0.65$ & $2.44 \pm 0.44$
\end{tabular}



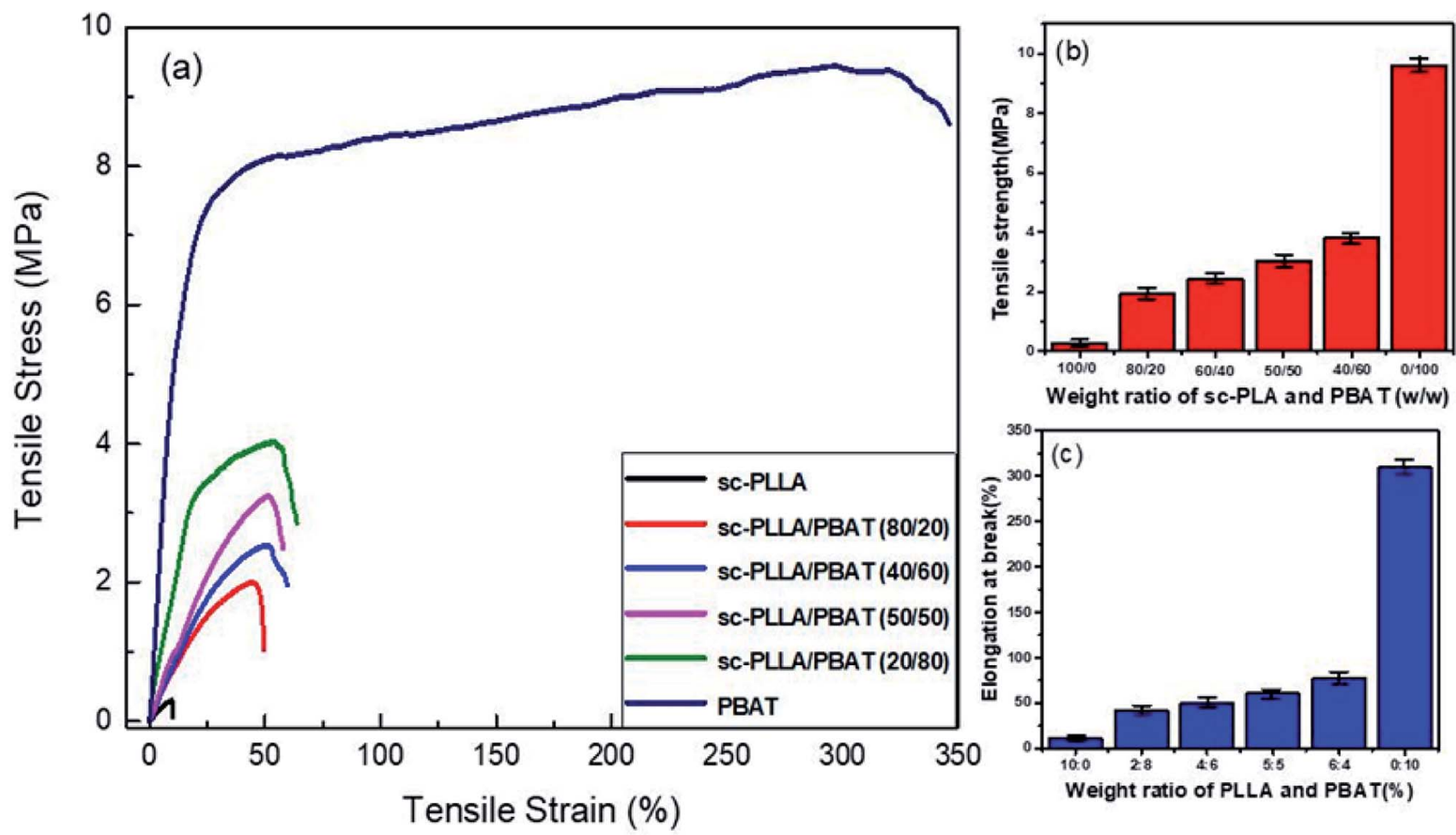

Fig. 6 (a) Stress-strain curves of sc-PLA/PBAT scaffolds; (b) plot of tensile and (c) strength elongation at break of sc-PLA/PBAT scaffolds with different PBAT concentration.

characteristic of ductile fracture become more obvious if the content of PBAT is higher than $80 \%$. The variation in tensile strength and elongation at break with varying weight ratio for sc-PLA/PBAT scaffolds is shown in Fig. 6b and c, respectively. There is a significant enhancement of tensile strength and elongation of scaffolds with increased content ratio of PBAT. The tensile strength for sc-PLA/PBAT scaffold with 50/50 weight ratio reached up to $3.02 \mathrm{MPa}$, which is an increase of 10 times of PLLA scaffold with 0.3 MPa seen at Fig. 6b. Surprisingly, the elongation at break also shows a similar trend with tensile

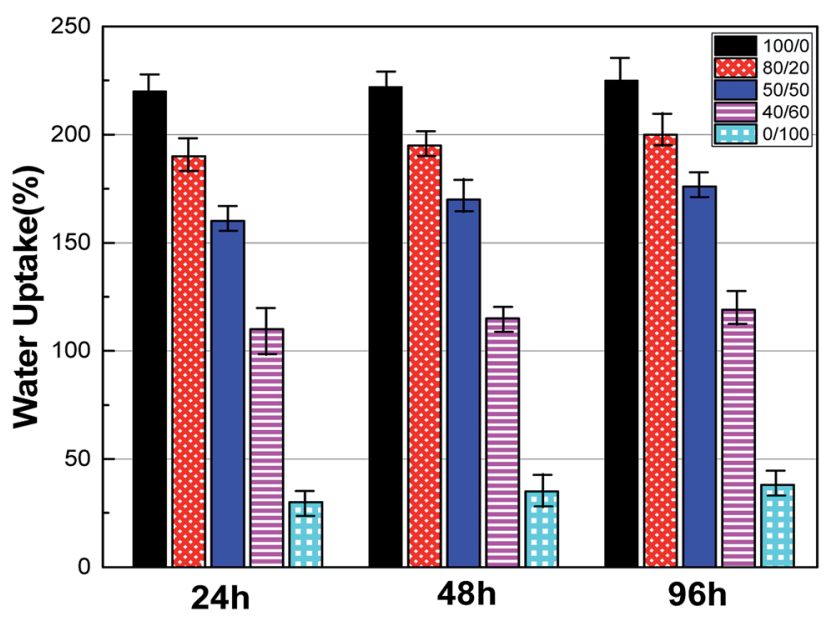

Fig. 7 Water uptake of Sc-PLA/PBAT scaffolds with different ratio (w) $W=100 / 0,80 / 20,50 / 50,40 / 60$ and 0/100) after 24, 48, and 96 hours soaking in PBS. strength (Fig. 6c). The enhanced mechanical properties are not only related to modifying effect by adding flexible PBAT into matrix, but also affected by the pore structures of scaffolds. As shown in Fig. 5 and Table 2, the addition of PBAT would lead to a lower porosity and smaller pore size for the porous sc-PLA/ PBAT scaffold. The smaller pore size is favorable for the strength enhancement, while the more PBAT is good for the increasing of toughness. For the sc-PLA/PBAT scaffold with a ratio $50: 50(\mathrm{w} / \mathrm{w})$, the elongation a break is 5 times higher than that of sc-PLA scaffold, and it still possesses an available toughness and maintains a highly opened porous structure. In other words, the two principles for PLLA used as tissue engineered cartilage, which is the scaffold material with both good elasticity for physiological function and pore formation for materials exchange of cells, can be tailored by the fabrication of porous PLA/PBAT scaffold.

\subsection{Wettability}

Wettability is a critical characteristic for application of scaffolds in tissue engineering. We measured the water uptake of scaffolds with porous structures by calculating the mass ratio after immersing them in PBS solution for different durations and comparing them with original mass. The water uptake results of scaffolds prepared with a series of weight ratio of sc-PLA and PBAT (100/0, 80/20, 50/50, 40/60 and 0/100) are shown in Fig. 7. The water uptake of the sample scaffolds represent relative stability for three different immersion durations, specifically 24 , 48, and 96 hours. However, the scaffold with higher porosity 


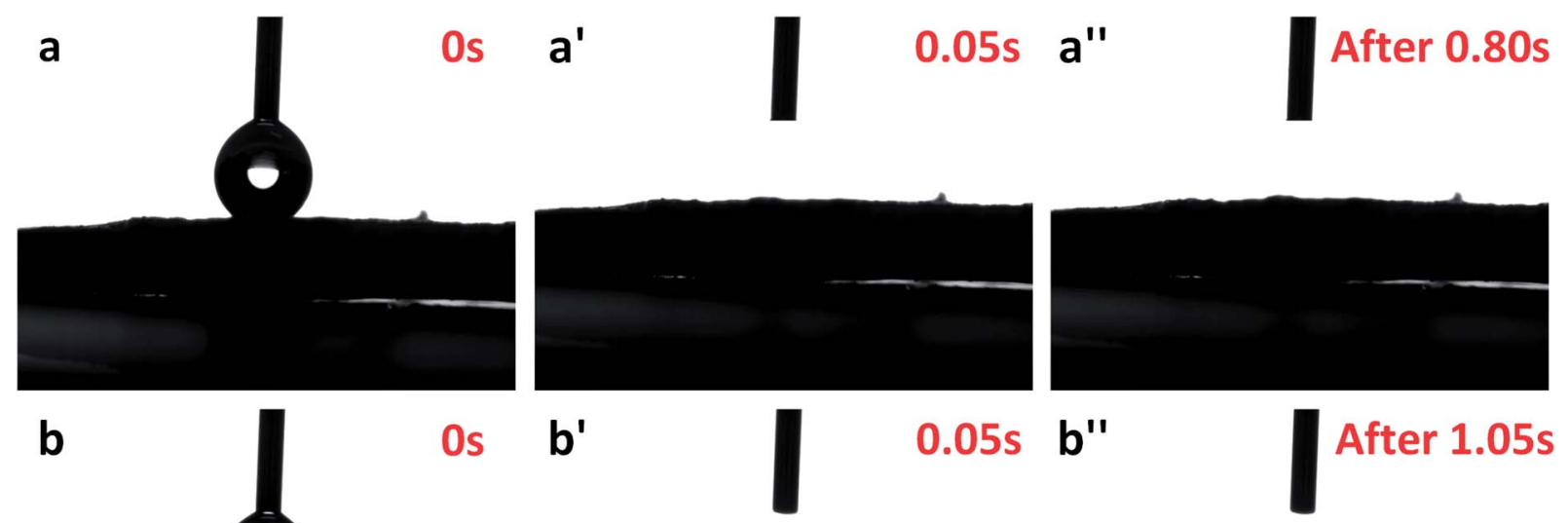

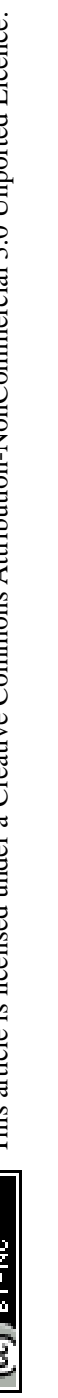

represents higher water absorption due to its more vacant sites. The maximum water absorption of sc-PLA scaffold rose to $225 \%$ with high porosity of $93.5 \%$. On the other hand, to understand the effect of PBAT content on the hydrophilicity of scaffolds, dynamic contact angle measurements were conducted. The photographs of sc-PLA scaffold, sc-PLA/PBAT scaffold (50/50), and imporous sc-PLA/PBAT membrane during the water absorption process are shown in Fig. 8. As can be seen, the drop water maintained stably on the sc-PLA/PBAT membrane after 3 minutes. However, the sc-PLA scaffold had absorbed a drop of

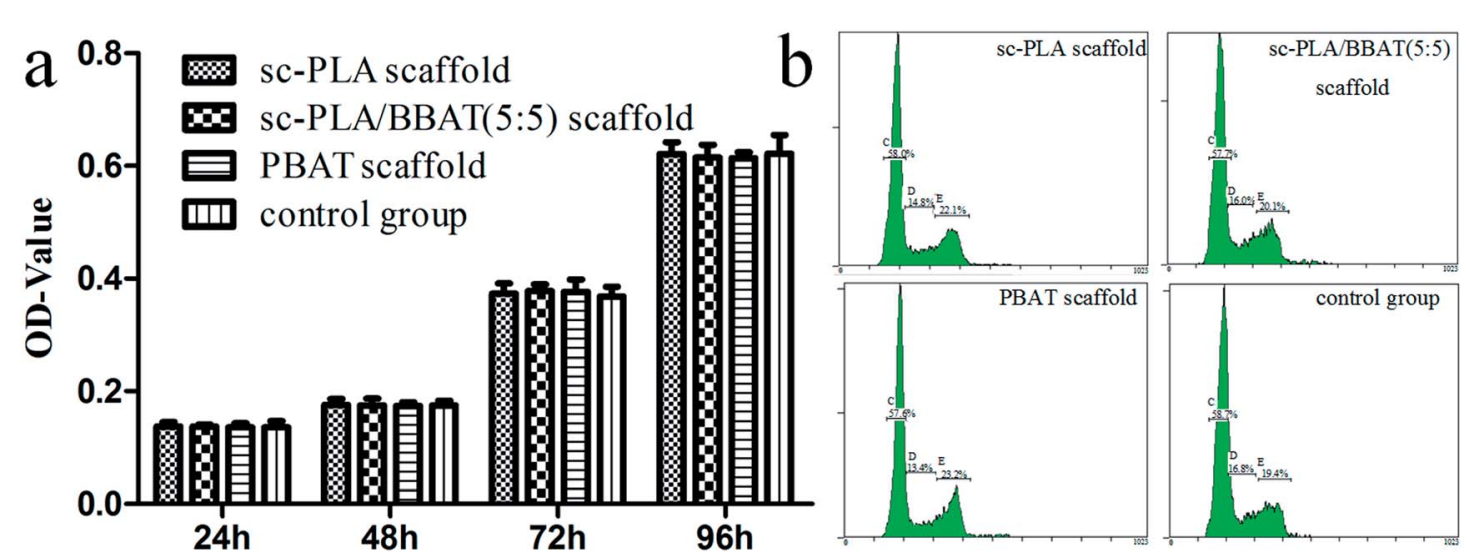

Fig. 9 Cell proliferation evaluated by FCM and MTT assays. (a) Survival rate tested by MTT assays, (b) cell cycle of L929 cells cultured on scaffolds detected by FCM. 

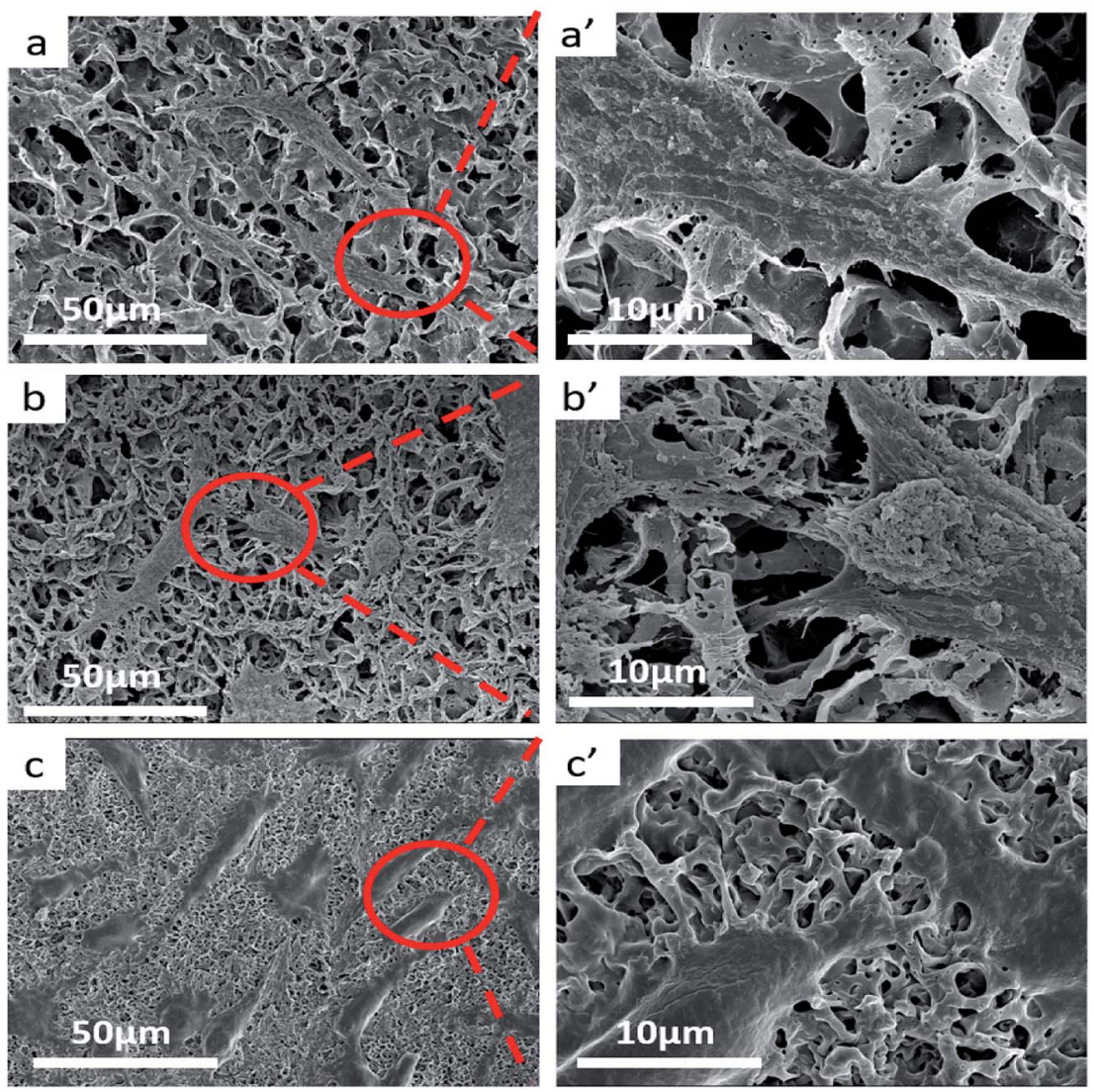

Fig. 10 SEM images of L929 cells cultured on sample scaffolds. (a-c) L929 cells cultured on sc-PLA, Sc-PLA/PBAT (50/50) and PBAT scaffolds, $\left(a^{\prime}-c^{\prime}\right)$ are higher magnification of the red square, respectively.

water in only $0.8 \mathrm{~s}$, and little longer $1.05 \mathrm{~s}$ of sc-PLA/PBAT scaffold. The water uptake of the scaffolds was significantly modified by introducing the open-porous pore structure and high porosity of scaffolds. Furthermore, the addition of flexible PBAT to the matrix results in thicker walls and decreases pore size and porosity as compared to those for neat PLA scaffold. The structural modifications may lead to interconnectivity reduction, which prevents water diffusion into the scaffolds and consequently, lower water absorption.

\subsection{Cell proliferation assay}

MTT assay was used to evaluate the survival rate of L929 cells cultured with sample scaffolds such as sc-PLA scaffold, sc-PLA/ PBAT (50/50) scaffold, and PBAT scaffold, respectively. As shown in Fig. 9a, the viability of L929 cells cultured with scaffolds was not significantly different from that in the control group cultured without scaffolds $(P>0.05)$. In order to further validate the effect of scaffolds on cell proliferation, cell cycle of each group was detected by FCM. The percentage of L929 cells in S stage was $14.87 \pm 0.12,16.13 \pm 0.15,13.50 \pm 0.10$, and $16.77 \pm$ 0.15 in sc-PLA scaffold, sc-PLA/PBAT (5/5) scaffold, PBAT scaffold, and control group, respectively (see Fig. 9b). According to cell proliferation index $(\mathrm{PI})=(\mathrm{S}+\mathrm{G} 2) \%$, the PI of sc-PLA scaffold, sc-PLA/PBAT (5/5) scaffold, PBAT scaffold, control group was $37.00 \pm 0.17,36.03 \pm 0.17,36.50 \pm 0.10$, and $36.53 \pm 0.42$, respectively. There was no significant difference between the experimental group and the control group $(P>0.05)$. These results indicate that the scaffold material has no significant effect on the cell proliferation activity.

\subsection{Cell growth and morphology}

SEM images suggest that sample scaffolds have good porous structure, and L929 cells were fully expanded, flattened, stretched out multiple pseudopods and microvilli, and attached closely to the surface of the material. The cells grew along the edge of the material and joined into pieces. In Fig. 10b', the pseudopodia of the cells can be clearly observed in the pore structure.

\section{Conclusions}

With the purpose of preparation of tissue engineering scaffolds of different organs, the scaffold material needs good elasticity for physiological function and pore formation for materials exchange of cells. In this work, PBAT as biodegradable, flexible material was introduced into PLA-based blend, and this system was fabricated with open-porous structure by non-solvent induced phase separation method. The porosity and openporous structure of sc-PLA/PBAT scaffolds could be tailored by controlling the ratio of dichloromethane and water phase. Furthermore, with the evidence from DSC, XRD and FTIR results, the formed PLA stereocomplex presented great effect on 
the open-porous structure. Compared to either PLLA/PBAT or PDLA/PBAT scaffold, the scaffold based on sc-PLA/PBAT presented sexangular pore structure and the most uniform walls thickness of about $1 \mu \mathrm{m}$. This uniform porous structure was strongly related to the intermolecular forces between PLLA and PDLA. Then the added PBAT into the sc-PLA blends showed important influence on the pore sizes, pore wall morphologies as well as mechanical properties of scaffolds. With the increase of PBAT concentration into the sc-PLA blends, the porous scPLA/PBAT scaffold presented a lower porosity and smaller pore size in consequence of increased tensile strength and also elongation-at-break. The wettability of our prepared porous scaffolds indicated the formed porous structure favorable for the water uptake, which is strongly related the pore size and porosity of the scaffold. Fibroblasts cells seeded on the structure maintained phenotypic shape, and the developed scaffold structure was observed to be highly capable of supporting the cell attachment and proliferation. The final sc-PLA/PBAT scaffolds possess high porosity, open-porous structure, enhanced mechanical properties, and excellent biocompatibility, which shows a great promise for its potential applications in tissue engineering.

\section{Conflicts of interest}

There are no conflicts to declare.

\section{Acknowledgements}

This work is funded by the National Natural Science Foundation of China (51773170). We thank Prof. Guang Cheng Zhang and XueTao Shi for providing laboratory facilities for the execution of this project.

\section{References}

1 L. Yi, S. Y. Xin, Y. J. Bian, Q. L. Dong, C. Y. Han, K. Xu and L. S. Dong, RSC Adv., 2015, 5, 24352-24362.

2 Y. Sun and C. He, ACS Macro Lett., 2012, 1, 709-713.

3 M. Orietta, M. Putti, L. Gardella, D. Cavallo, A. Basso, M. Prato and S. Nitti, Macromolecules, 2014, 47, 4718-4727.

4 G. Arvind and V. Katiyar, ACS Sustainable Chem. Eng., 2017, 5, 6835-6844.

5 A. Michalski, T. Makowski, T. Biedroń, M. Brzeziński and T. Biela, Polymer, 2016, 90, 242-248.

6 Z. J. Xiong, X. Q. Zhang, R. Wang, S. Vos, R. Y. Wang, C. A. Joziasse and D. J. Wang, Polymer, 2015, 76, 98-104.

7 Z. Jing, X. Shi and G. Zhang, Polymer, 2017, 121, 124-136.

8 Z. Jing, X. Shi and G. Zhang, Polymer, 2016, 92, 210-221.

9 S. Zeng, Z. Cui, Z. Yang, J. Si, Q. Wang, X. Wang, K. Peng and W. Chen, J. Mater. Sci., 2016, 51, 9958-9970.

10 Z. Xiong, F. Liu, A. Gao, H. Lin, X. Yu and Y. Wang, RSC Adv., 2016, 6, 20492-20499.
11 S. Hua, F. Chen, Z. Liu, W. Yang and M. Yang, RSC Adv., 2015, 6, 32-49.

12 J. B. Zeng, K. A. Li and A. K. Du, $R S C A d v ., 2015$, 5, 3254632565.

13 Q. Q. Yao, J. G. Cosme, T. Xu, J. M. Miszuk, P. H. Picciani, H. Fong and H. L. Sun, Biomaterials, 2017, 115, 115-127.

14 Y. S. Nam and T. G. Park, J. Biomed. Mater. Res., 1999, 1, 8-17. 15 O. Qutachi, J. R. Vetsch, D. Gill, H. Cox, D. J. Scurr, S. Hofmann, R. Müller, R. A. Quirk, K. M. Shakesheff and C. V. Rahman, Acta Biomater., 2014, 10, 5090-5098.

16 Y. Di, S. Iannace, E. D. Maio and L. Nicolais, Macromol. Mater. Eng., 2005, 290, 1083-1090.

17 W. Zhao, J. Li, K. Jin, W. Liu, X. Qiu and C. Li, Mater. Sci. Eng., C, 2016, 59, 1181-1194.

18 J. Gu, Y. Guo, Z. Lv, W. Geng and Q. Zhang, Composites, Part A, 2015, 78, 95-101.

19 J. Gu, Q. Zhang, J. Dang, C. Yin and S. Chen, J. Appl. Polym. Sci., 2012, 124, 132-137.

20 T. Patrício, M. Domingos, A. Gloria and P. Bártolo, Procedia CIRP, 2013, 5, 110-114.

21 R. Scaffaro, F. Lopresti, L. Botta, S. Rigogliuso and G. Ghersi, J. Mech. Behav. Biomed. Mater., 2016, 54, 8-20.

22 A. M. Haaparanta, E. Järvinen, I. F. Cengiz, V. Ellä, H. T. Kokkonen, I. Kiviranta and M. Kellomäki, J. Mater. Sci.: Mater. Med., 2014, 25, 1129-1136.

23 X. Shi, G. Zhang, Y. Liu, Z. Ma, Z. Jing and X. Fan, Polym. Adv. Technol., 2016, 27, 550-560.

24 L. Jiang, M. P. Wolcott and J. Zhang, Biomacromolecules, 2006, 7, 199-207.

25 F. Signori, A. Boggioni, M. C. Righetti, C. E. Rondan, S. Bronco and F. Ciardelli, Macromol. Mater. Eng., 2015, 300, 153-160.

26 L. C. Lins, S. Livi, J. Duchet-Rumeau and J. F. Gérard, RSC Adv. , 2015, 5, 59082-59092.

27 R. Al-Itry, K. Lamnawar and A. Maazouz, Polym. Degrad. Stab., 2012, 97, 1898-1914.

28 R. Al-Itry, K. Lamnawar and A. Maazouz, Eur. Polym. J., 2014, 58, 90-102.

29 H. Tsuji, S. Yamamoto, A. Okumura and Y. Sugiura, Biomacromolecules, 2010, 11, 252-258.

30 R. Herrera, L. Franco, A. Rodriguez-Galan and J. Puiggali, J. Polym. Sci., Part A: Polym. Chem., 2002, 40, 4141-4157.

31 Y. Sun and C. He, RSC Adv., 2013, 3, 2219-2226.

32 Z. Jing, X. Shi, G. Zhang and R. Lei, Polym. Int., 2015, 64, 1399-1407.

33 Y. Sun and C. He, ACS Macro Lett., 2012, 1, 709-713.

34 Z. Jing, X. Shi, G. Zhang and J. Li, Polym. Adv. Technol., 2015, 26, 528-537.

35 L. C. Arruda, M. Magaton, R. E. Bretas and M. M. Ueki, Polym. Test., 2015, 43, 27-37. 